\title{
A Compact Asymmetrically Slotted Antipodal Vivaldi Antenna for MIMO Imaging Systems.
}

\section{Djamila ZIANI}

Universite Abou Bekr Belkaid Tlemcen

Sidi Mohammed MERIAH ( $\nabla$ meriah_m@yahoo.com )

Universite Abou Bekr Belkaid Tlemcen https://orcid.org/0000-0003-0010-2296

Foued DERRAZ

Universite Abou Bekr Belkaid Tlemcen

\section{Lotfi MERAD}

Universite Abou Bekr Belkaid Tlemcen

\section{Research Article}

Keywords: Ultra-wide band, Antipodal Vivaldi antenna, Microwave imaging, Time domain analysis

Posted Date: October 22nd, 2021

DOI: https://doi.org/10.21203/rs.3.rs-906609/v1

License: (c) (i) This work is licensed under a Creative Commons Attribution 4.0 International License. Read Full License 


\title{
A Compact Asymmetrically Slotted Antipodal Vivaldi Antenna for MIMO Imaging Systems.
}

\author{
Djamila ZIANI • Sidi Mohammed MERIAH • Fouad DERRAZ • Lotfi \\ MERAD
}

Received: date / Accepted: date

\begin{abstract}
In this paper, we propose a Compact Asymmetrically Slotted Antipodal Vivaldi Antenna (CASAVA) design for MIMO imaging systems. The structure has the ability to extend the antenna bandwidth in low-end frequencies achieving a fractional bandwidth of $123,32 \%(4.743-20 \mathrm{GHz})$. Good results are obtained in term of return loss, radiation pattern and gain. A time-domain study has been also performed to characterize the antenna behavior in case of an UWB pulse is used. To further validate our design, an application for MIMO imaging system is also proposed and evaluated. The antenna performances demonstrate that it is a good candidate for microwave imaging applications.
\end{abstract}

Keywords Ultra-wide band · Antipodal Vivaldi antenna $\cdot$ Microwave imaging $\cdot$ Time domain analysis

\section{Introduction}

Recently, active microwave imaging has become of a great interest in both academic and industrial communities. It is widely used in several applications for detecting and locating objects [1],[2],[3]. For better images, high resolution is required in these imaging systems which can be theoretically enhanced by a large aperture and a wide factional bandwidth [4]. For that reason, many studies had substantiated that MIMO arrays combined with ultra-wideband (UWB) technology -with a fractional bandwidth of more than $20 \%$ - is the perfect option not only for high resolution but also for the ability of its signals to penetrate in different optically opaque materials. For such systems, designing a

D. ZIANI, S.M. MERIAH, F. DERRAZ, L. MERAD

Laboratory of Telecommunications Tlemcen (LTT), Faculty of Technology, University of Tlemcen, Algeria

E-mail: ziani.djamilla@gmail.com suitable antenna is a crucial step. In general, the antennas should have a wide fractional bandwidth, small size, simple, and low cost of fabrication [2]. Beside these requirements, gain and radiation pattern stability, low signals distortion have to be achieved. Hence Vivaldi antennas have always been the most suitable thanks to their wide fractional bandwidth, small size, simplicity, and cost-free [5]. Besides that, gain and radiation pattern stability, low signals distortion are also achieved.

The conventional Vivaldi tapered slot antenna (TSA) was firstly introduced by Gibson in 1979 [6]. However, the TSA presents some limitations in term of bandwidth and feeding complexity. Few years later, Gazit has proposed a new family of the Vivaldi antennas (Antipodal Vivaldi Antenna: AVA) [7]. The AVA is proposed to solve both the bandwidth limitation and the feeding problem in coplanar ones by eliminating the complexity of the balun. Obviously, in the Antipodal Vivaldi Antenna, one of the material layers is printed on top and the other one is printed on the bottom of the dielectric substrate material. This antenna can be fed easily by micro-strip line, soldering the connector to the two sides of the PCB material. In the literature, many kinds of Vivaldi antennas have been investigated for different aims [8],[9],[10], [11],[12]. However, even if the AVA antennas can achieve high frequency end of the operating band, the low-end frequency is still limited. Thus, Moosazadeh et al [13] have proposed an elliptically-tapered antipodal Vivaldi antenna where the lowfrequency is extended to $1.65 \mathrm{GHz}$ using combshape slits. In [14], a Koch fractal slots are used in the design of the AVA to improve the antenna frequency band for medical microwave imaging applications. Another antipodal Vivaldi antenna is proposed in [15] using a triangular slits and bending the inner edges of the top and bottom radiators to extend the lower cut- 
off frequency (which is extended to $2 \mathrm{GHz}$ ) and enhance the antenna gain. Although, dimension of these antennas are relatively large $(\geq 100 \mathrm{~mm})$ which limits their use in MIMO array systems for microwave imaging applications.

In many studies, the designs rely on the symmetry of the two faces of the AVA antenna. In our work, we propose a small antipodal Vivaldi antenna where the concept involves introducing slots asymmetrically in both sides of the antenna expecting to further enhance its performances. The compact asymmetrically slotted AVA (CAS-AVA) based on a conventional antenna design can extend the lower operating frequency from $8 \mathrm{GHz}$ to $4.74 \mathrm{GHz}$.

\section{Antenna design}

We consider a conventional Basic AVA (see Fig. 1) with dimensions of $24 \times 33 \mathrm{~mm}^{2}$ printed on a FR4 substrate with a dielectric permittivity of $\epsilon_{r}=4.3$, and thickness $h=1.5 \mathrm{~mm}$. The metallization of both faces is of a thickness $t=0.035 \mathrm{~mm}$. A 50-Ohm micro-strip line will feed the antenna. The tapers of the Vivaldi antenna are defined accordingly to an exponential profile given by the equations below:

$y=c_{1} \cdot \exp ^{(R x)}+c_{2}$

$c_{1}=\frac{y_{2}-y_{1}}{\exp ^{R x_{2}}-\exp ^{R x_{1}}}$

$c_{2}=\frac{y_{1} \exp ^{R x_{2}}-y_{2} \exp ^{R x_{1}}}{\exp ^{R x_{2}}-\exp ^{R x_{1}}}$

Where $R$ presents the opening rate of the exponential taper and $c 1, c 2$ are constants defined by equations 2 and $3,(x 1, y 1)$ and $(x 2, y 2)$ are the starting and ending points of the taper curve respectively. Numerical simulations are done the electromagnetic simulator CST MWS software (Computer Simulation Technology- Microwaves Studio) [16]

It is worth to note that the B-AVA impedance matching cannot achieve a frequency lower than $7.98 \mathrm{GHz}$ (Fig. 2). For that reason, we have exploited the advantage of inserting slots in the interest of widening the antenna bandwidth. Firstly, three slots are introduced symmetrically in both top and bottom faces of the antenna. However, we can notice from Fig. 2 that no significant improvement can be observed with symmetric slots.

Conducted by this drawback, we have modified the conventional concept shape of the AVA to an asymmetric form. We present in Fig. 3 the final design of the

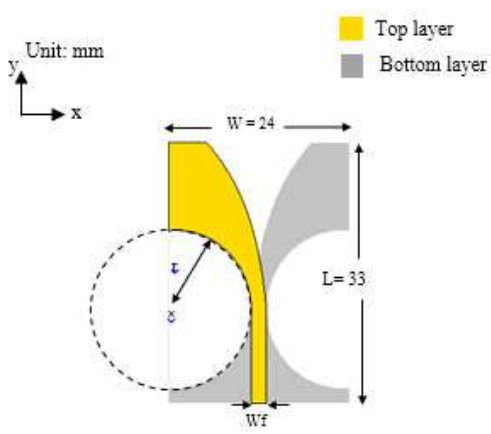

Fig. 1: Basic Antipodal Vivaldi Antenna (B-AVA) design

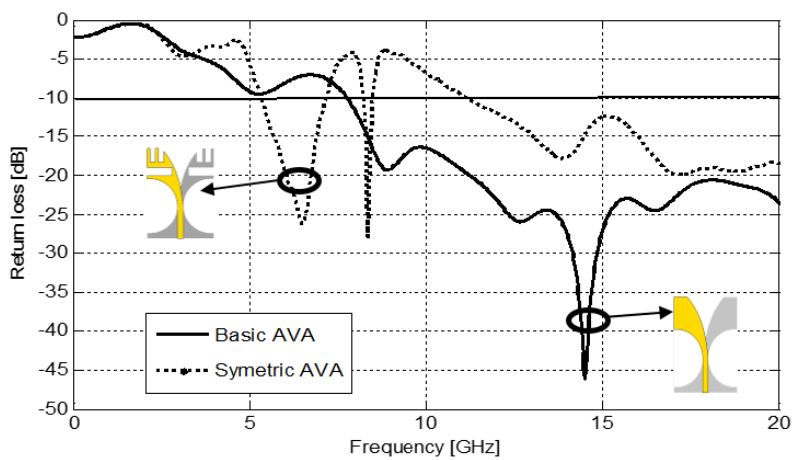

Fig. 2: Return loss of the B-AVA and symmetric slotted AVA

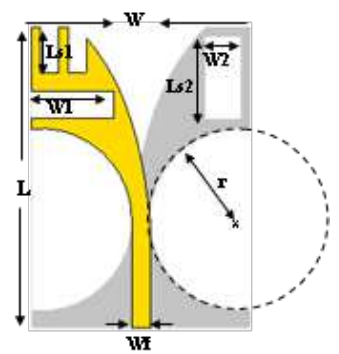

Fig. 3: Proposed design of the Compact Asymmetric Slotted Antipodal Vivaldi Antenna (AS-AVA)

proposed Compact Asymmetrically Slotted AVA (CASAVA), where we kept the three slots in the antenna top while introducing a single rectangular slot in the antenna bottom.

\section{Results and discussions}

In this section, we will discuss the antennas results in frequency domain, the discussion concern return loss behavior, current distribution, radiation pattern, gain and efficiency. 


\subsection{Return loss}

To fix the slots dimensions, several parametric studies are conducted on the parameters $W_{1}, L_{s 1}, W_{2}$ and $L_{s 2}$ of the top and bottom slots. For the top slots we present in Fig. 4 and Fig.5 the effects of the width $\mathrm{W} 1$ and larger Ls1 on the return loss parameter. The introduction of these slots has considerably enhance the impedance matching in low frequencies except in a slight frequency range from $7.43 \mathrm{GHz}$ to $8.07 \mathrm{GHz}$. The chosen values are $W 1=9 \mathrm{~mm}$ and $L s 1=5 \mathrm{~mm}$.

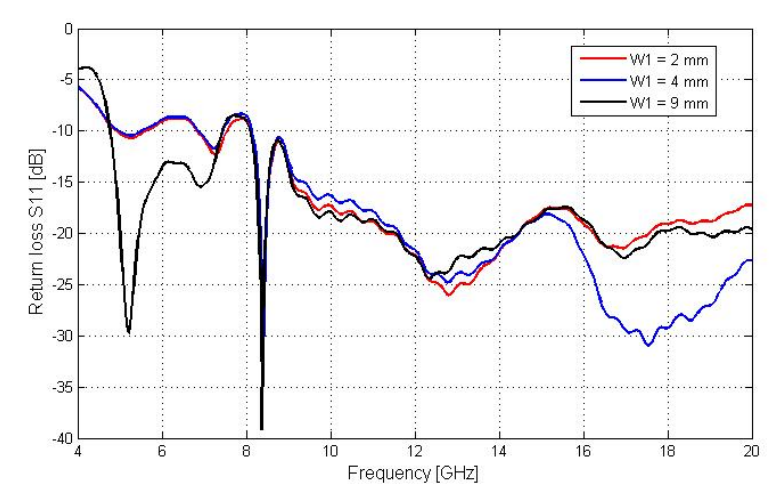

Fig. 4: Effect of the slot width $W_{1}$ with $L_{s 1}=2 \mathrm{~mm}$

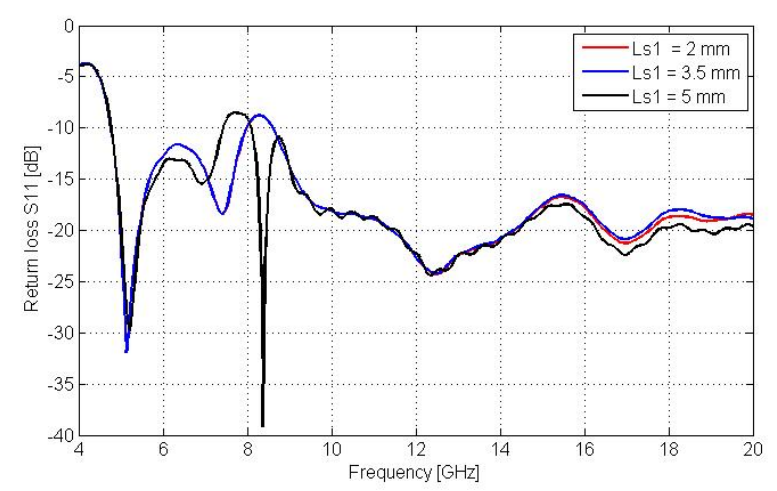

Fig. 5: Effect of the slot larger $L_{s 1}$ with $W_{1}=9 \mathrm{~mm}$.

For the bottom slot, we present in Fig.6, the effect of the slot width $\left(W_{2}\right)$ on the antenna impedance matching. It is clearly shown that better results are obtained for $W_{2}=3 \mathrm{~mm}$. Regarding the slot larger, the Fig. 7 presents its influence on the return loss parameter. The antenna matching is improved for the value $L_{s 2}=9 \mathrm{~mm}$.

The impedance matching of the proposed CAS-AVA compared with the B-AVA and the symmetric AVA is

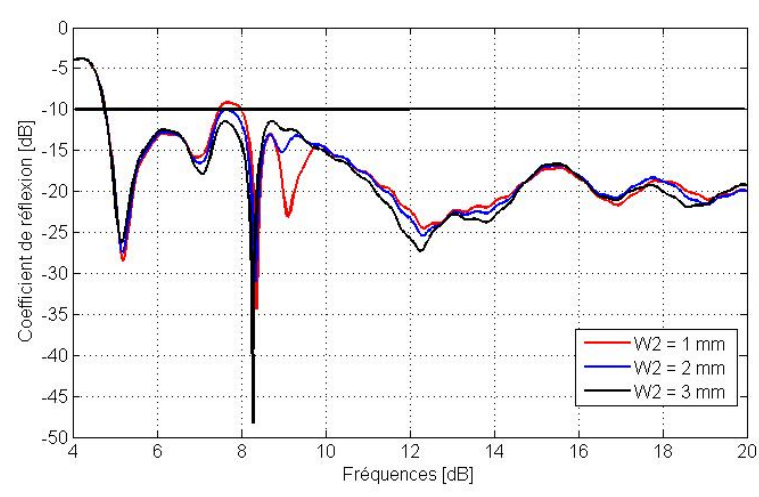

Fig. 6: Effect of the slot width $W_{2}$ with $W_{1}=9 \mathrm{~mm}$, $L_{s 1}=5 \mathrm{~mm}$ and $L_{s 2}=1 \mathrm{~mm}$.

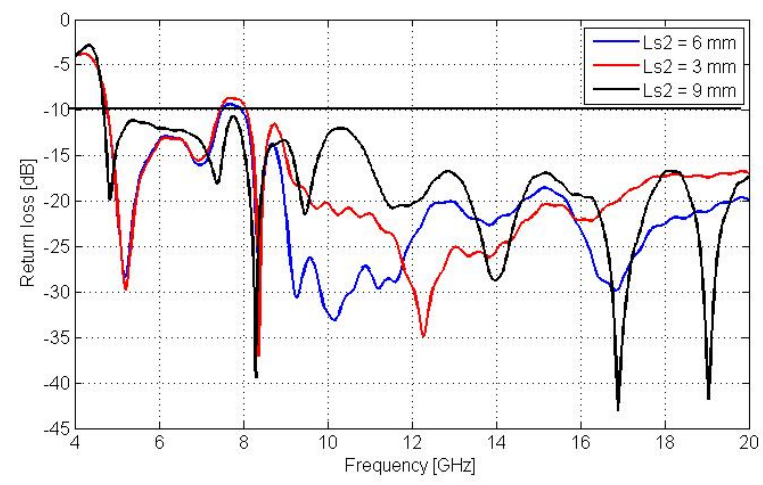

Fig. 7: Effect of the slot larger $L_{s 2}$ with $W_{1}=$ $9 \mathrm{~mm}, L_{s 1}=3 \mathrm{~mm}$ and $W_{2}=3 \mathrm{~mm}$.

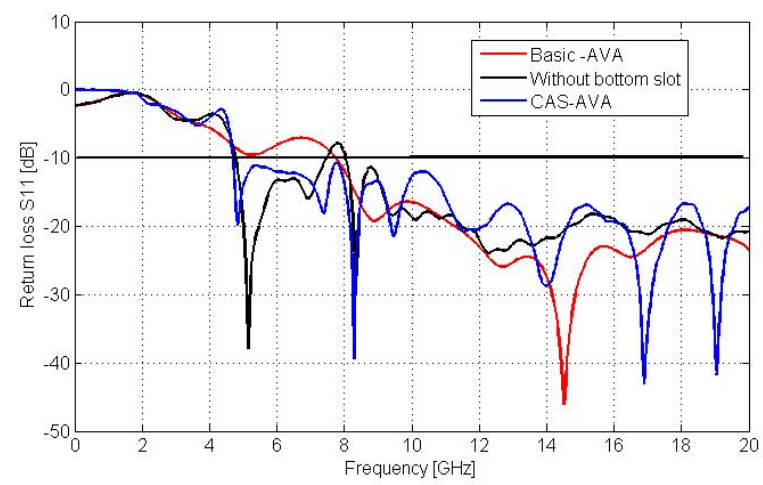

Fig. 8: Basic AVA, Basic AVA without bottom slot and CAS-AVA return losses

depicted in Fig. 8. The CAS-AVA lower frequency can be extended to the limit of $4.74 \mathrm{GHz}$. No upper limit has been found before $20 \mathrm{GHz}$ with a fractional bandwidth of more than $123 \%$. Final parameters are listed in Table 1. 
Table 1: Optimized dimensions of the CAS-AVA

\begin{tabular}{ll}
\hline Variable & Dimension $(\mathrm{mm})$ \\
\hline $\mathrm{W}$ & 24 \\
$\mathrm{~L}$ & 33 \\
$W_{f}$ & 2 \\
$W_{1}$ & 9 \\
$W_{2}$ & 3 \\
$L_{s 1}$ & 5 \\
$L_{s 2}$ & 9 \\
$\mathrm{r}$ & 10 \\
\hline
\end{tabular}

\subsection{Current distribution}

In Fig. 9 the current distributions of the CAS-AVA at several frequencies $4.9 \mathrm{GHz}, 7 \mathrm{GHz}$ and $10 \mathrm{GHz}$ are illustrated. It is clearly noticed that the current density is higher around the lower slot region in low frequencies and take a high level around upper slots in high frequencies. So that, additional path for surface current is created with the slots introduction. That leads to create new resonances as confirmed in Figure 8 compared to the B-AVA.

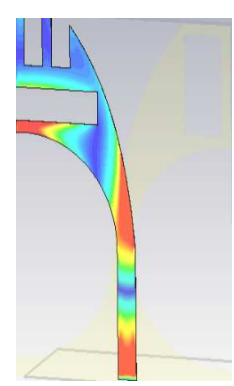

(a) $4.7 \mathrm{GHz}$

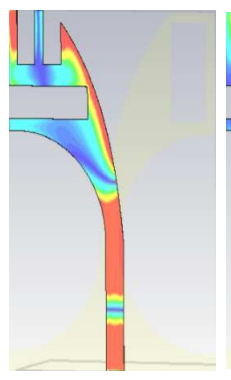

(b) $7 \mathrm{GHz}$

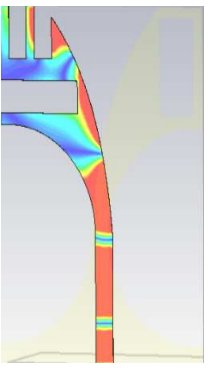

(c) $10 \mathrm{GHz}$

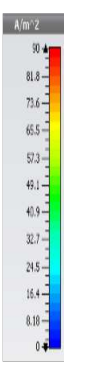

Fig. 9: Current distributions on the proposed antenna at different frequencies

\subsection{Radiation pattern}

Both $\mathrm{E}$ and $\mathrm{H}$ plane radiation patterns of the proposed antenna are plotted in Fig.10 at several frequencies notably $5 \mathrm{GHz}, 11 \mathrm{GHz}$ and $16 \mathrm{GHz}$. As we can see, the antenna presents a directional radiation patterns at all mentioned frequencies. Worth noticing that, the CASAVA is more directive in E plane than $\mathrm{H}$ plane

\subsection{Gain and effeciency}

The realized gain vs frequencies of the CAS-AVA; is shown in Fig. 11. In the operational frequency band,

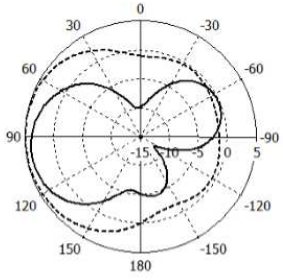

(a) $5 \mathrm{GHz}$

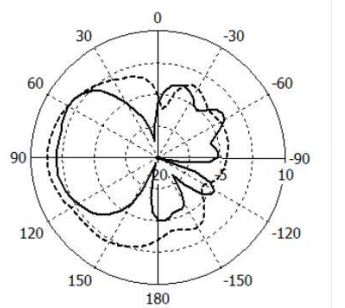

(c) $16 \mathrm{GHz}$

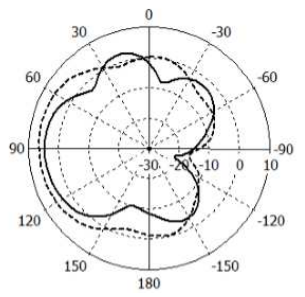

(b) $11 \mathrm{GHz}$
- E plane (xoy)

---- H plane (zoy)
Fig. 10: 2-D radiation pattern for $\mathrm{E}$ and $\mathrm{H}$ planes at different frequencies

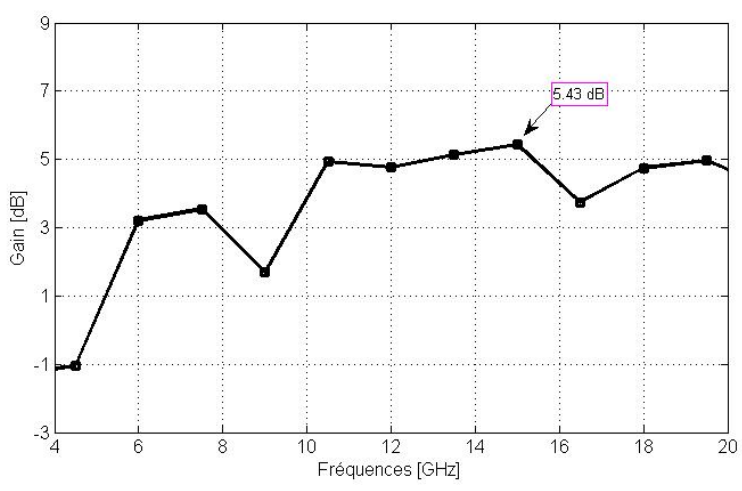

Fig. 11: Antenna gain vs frequencies

good gain values are observed and the maximum gain is picked at $15 \mathrm{GHz}$ with a value of $5.43 \mathrm{~dB}$. For our application, this gain is considered satisfactory given the proximity of the antenna and objects to explore.

The radiation efficiency is a primordial parameter as it measures the radiated power with respect to the power injected to the antenna. For our proposed antenna, the radiation efficiency remains above $70 \%$ in the entire operating frequency band as illustrated in Fig. 12 .

\section{Time domain analysis}

In microwave imaging applications, evaluating the time domain behaviour and studying the dispersion is a crucial step for UWB antennas [17]. It can be estimated with the measurement of signal distortion through trans- 


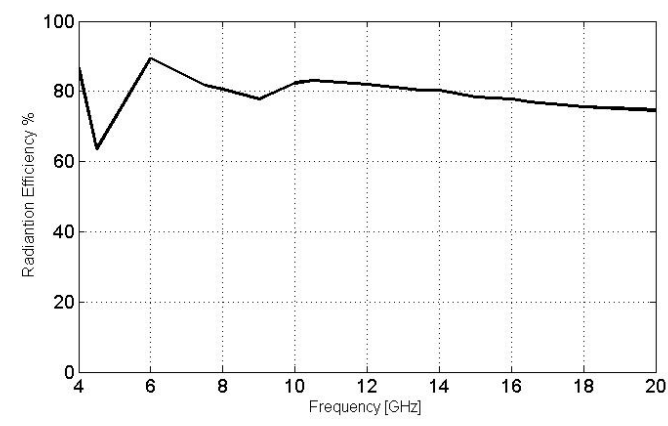

Fig. 12: Antenna efficiency vs frequencies

fer function, group delay parameter [18] and system fidelity factor SFF [19]. For that, time domain analysis of the proposed antenna is carried out by considering a system of two identical CAS-AVA antennas (See Fig. 13). The two CAS-AVA are separated by distance noted $\mathrm{d}=10 \mathrm{~cm}$. This is needed to have the antennas in the far-field region of each other. The antennas are placed in two different orientations, face orientation (see Fig. 13a) and side orientation (see Fig. 13b). One of the two antennas is assumed to be the transmitter and the other one acts as a receiver. The transmitter CAS-AVA generate the Gaussian's 5th derivative pulse[20] shown in Fig. 14, since it's spectrum meets the FCC ${ }^{1}$ mask. For our application we have used the numerical model of Gaussian derivative given by the relation below:

$y(t)=A\left(\frac{-t^{5}}{\sqrt{2 \pi} \sigma^{11}}+\frac{10 t^{3}}{\sqrt{2 \pi} \sigma^{9}} \frac{-15 t}{\sqrt{2 \pi} \sigma^{7}}\right) \cdot \exp \left(\frac{-t^{2}}{2 \sigma^{2}}\right)$

Where $\mathrm{A}$ is a constant chosen to meet the limitations set by the FCC. To cover the right frequency band, the value of $\sigma$ was set at 50.788 ps.

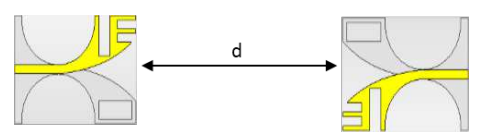

(a) Face orientation

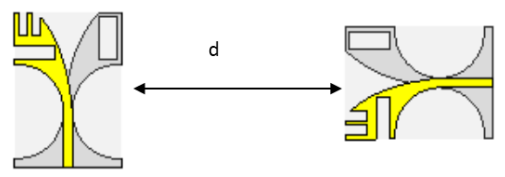

(b) Side orientation

Fig. 13: Antennas orientations

To achieve a transmission process without significant distortion, the transfer function $\left(S_{21}\right)$ magnitude

1 Federal Communications Commission

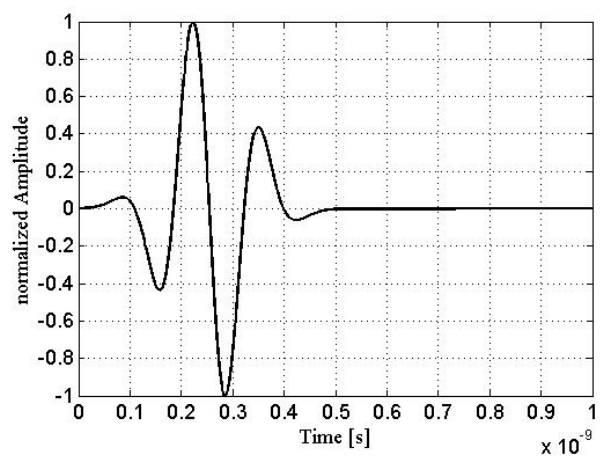

Fig. 14: Time domain representation of the Gaussian 5 th derivative
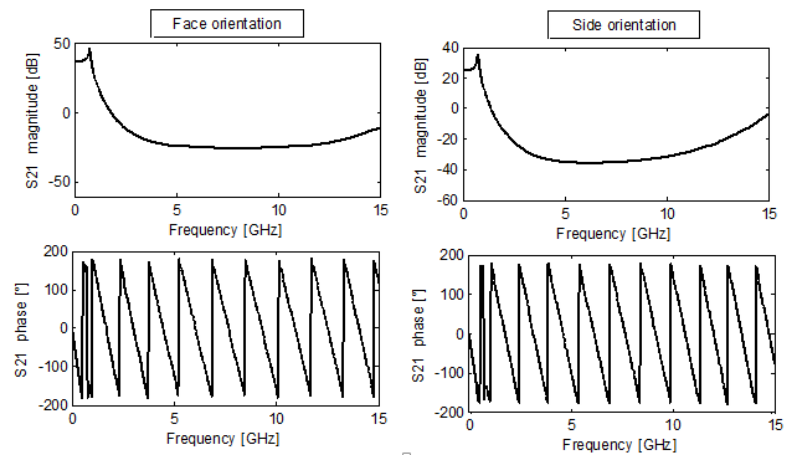

Fig. 15: Transfer's function magnitude and phase for Face orientation and Side orientation

and phase should be respectively flat and linear. We have drawn in Fig. 15 both magnitude and phase of $S_{21}$, in the entire frequency band they presents a good performance ensuring a best transmission with less distortion.

As well, a flat group delay parameter is also required, and mathematically it can be evaluated through the transfer's function phase by 5 .

Groupe delay $=-\frac{d \phi}{d f}$

Where $\phi$ is the $S_{21}$ phase. A constant group delay results to in linear $S_{21}$ phase. It can be seen from the Fig. 16, which presents the simulated group delay that it is around $0.6 \mathrm{~ns}$ and is quiet flat in the entire operating frequency band with variation of less than $1 \mathrm{~ns}$. The system fidelity factor is an important parameter for quantifying distortion; it judges the resemblance between the transmitted and received signals. A value less than $50 \%$ of the SFF will deliver a high distortion. It can be calculated using 6 .

$S F F=\max \frac{\int_{-\infty}^{\infty} S_{t}(t) \cdot S_{r}(t-\tau) d t}{\sqrt{\int_{-\infty}^{\infty}\left|S_{t}(t)\right|^{2} \cdot \int_{-\infty}^{\infty}\left|S_{r}(t)\right|^{2} d t}}$ 


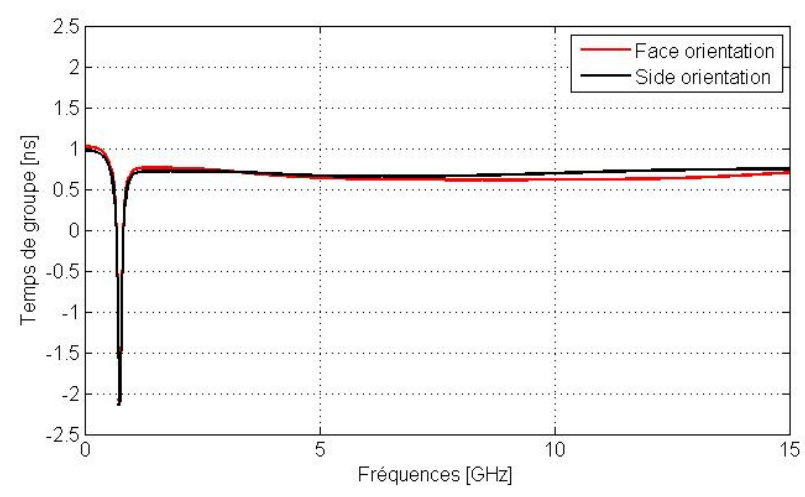

Fig. 16: Group delay vs frequencies
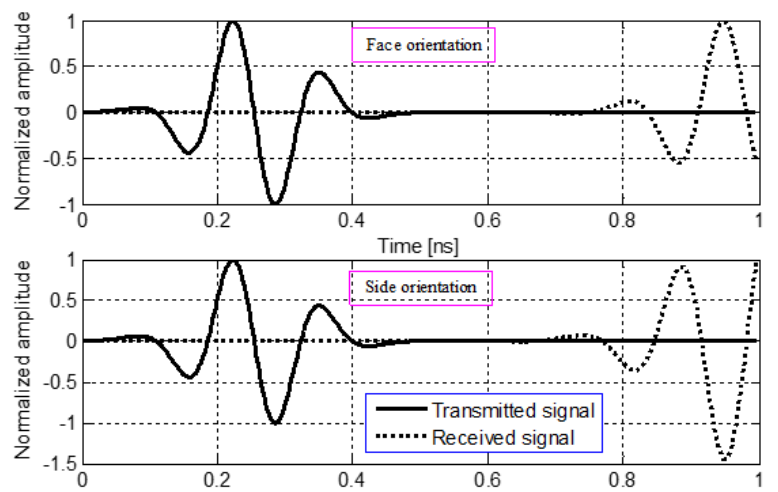

Fig. 17: Normalized transmitted and received signals

Table 2: System fidelity factor

\begin{tabular}{ll}
\hline Orientation & fidelity values \\
\hline Face orientation & $89.93 \%$ \\
Side orientation & $92.65 \%$ \\
\hline
\end{tabular}

$S_{t}(t)$ and $s_{r}(t)$ are the transmitted and received signals respectively. The normalized transmitted and received time signals are illustrated in Fig. 17. The SFF results for the two different orientations are summarized in Table 2. We notice that good values are achieved, in other words, the proposed antenna will not seriously distort the transmitted signal.

\section{Experimental results}

In order to verify the impedance matching of design, the proposed antenna has been fabricated (Fig. 18), fed by a $50 \Omega$ SMA connector and tested with ANRITSU MS2037C VNA. It is clearly shown from the Fig. 19 the agreement between the simulated results and the ones reached by the measurement. At high frequencies
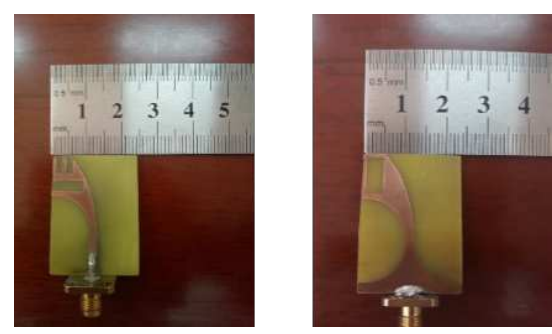

Fig. 18: Fabricated prototype of the CAS-AVA (left: top view and right: bottom view)

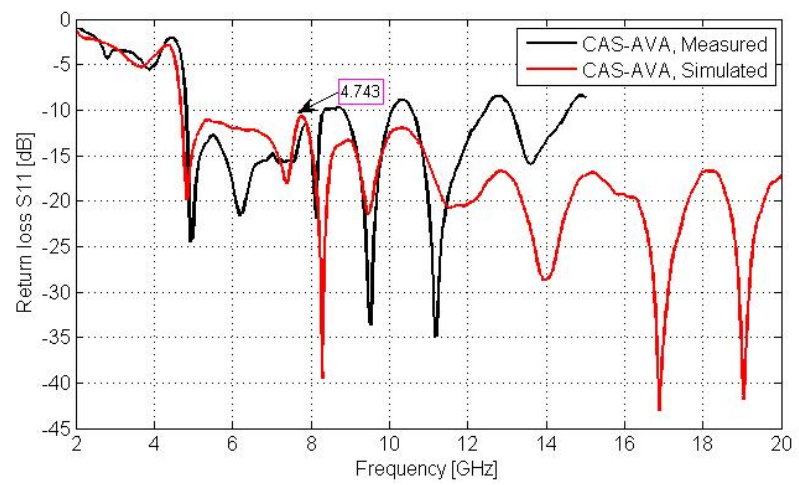

Fig. 19: Measured and simulated return loss of the proposed antenna (CAS-AVA)

$(\geq 10 \mathrm{GHz}$ ), the degradation is possibly caused by the substrate dielectric materials on signal loss of the microstrip line.

Table 3 summarizes characteristicsin terms of dimensions and bandwidth of others designed antennas in recent related work. As seen, reported antennas can achieve a wide bandwidth at the expense of large dimensions whileour design can fulfill a satisfactory tradeoff between size and large bandwidth.

\section{MIMO imaging system with the proposed CAS-AVA}

In the interest of demonstrating the designed antenna validity for microwave imaging, we designed a MIMO array using CAS-AVAwith a 2D topology as shown in Fig.20.

Designed under CST MWS software which allows 3D simulations, the model of this simulation includes the antenna array as well as a metallic non-regular target as presented in Fig.21. The largest dimension of the target being $100 \mathrm{~mm}$ and it is placed at a distance $\mathrm{d}=$ $5 \mathrm{~cm}$ from the array.

Identical antennas are used in $\mathrm{Tx}$ and $\mathrm{Rx}$ arrays with a fractional bandwidth greater than $123 \%$, as well the same UWB impulse is used for all transmitters 
Table 3: System fidelity factor

\begin{tabular}{|c|c|c|c|c|}
\hline Ref. & Dimensions & Dielectric & \multicolumn{2}{|c|}{ Bandwidth } \\
\hline 8 & $44 \times 49$ & 2.2 & $(8-18)$ & $\mathrm{GHz}$ \\
\hline & & & $76.92 \%$ & \\
\hline 9 & $100 \times 100$ & 4.4 & $(1-35)$ & $\mathrm{GHz}$ \\
\hline & & & $189 \%$ & \\
\hline 10 & $42.8 \times 57.3$ & 4.4 & (3.6-10) & $\mathrm{GHz}$ \\
\hline 11 & $50 \times 56$ & 4.4 & $\begin{array}{l}94.11 \% \\
(2-12) \\
143 \%\end{array}$ & $\mathrm{GHz}$ \\
\hline 12 & $36.3 \times 59.81$ & 6.15 & $\begin{array}{l}(5.08-11) \\
73.63 \%\end{array}$ & $\mathrm{GHz}$ \\
\hline 13 & $120 \times 202$ & 3.38 & $\begin{array}{l}(1.65-18) \\
166.41 \%\end{array}$ & $\mathrm{GHz}$ \\
\hline 14 & $36.3 \times 59.81$ & 6.15 & $\begin{array}{l}(4.87-11) \\
77.25 \%\end{array}$ & $\mathrm{GHz}$ \\
\hline Our work & $24 \times 33$ & 4.3 & $\begin{array}{l}(4.743-20) \\
\text { GHz } 123 .\end{array}$ & $32 \%$ \\
\hline
\end{tabular}

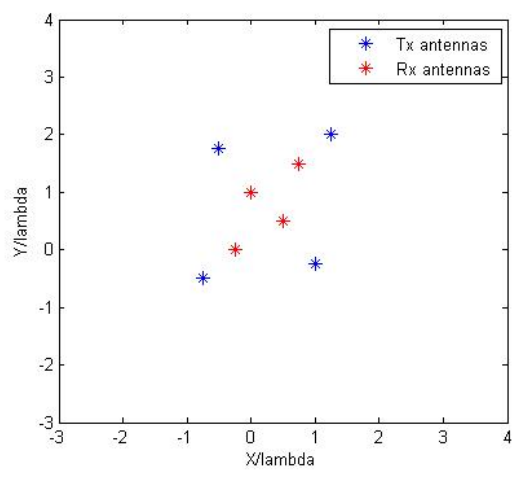

Fig. 20: 2D UWB MIMO array topology

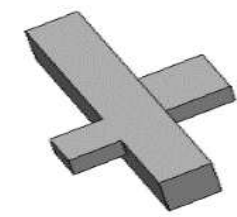

Fig. 21: Target shape

(Fifth Gaussian derivative). The scattered signals are received in term of transfer functions in frequency domain over all the frequency band. Then, they are transferred into time domain by the inverse Fourier Transform. The gathered data from all $\mathrm{Tx} / \mathrm{Rx}$ pairs are processed using Back Projection (BP) imaging algorithm [1] to reconstruct a three dimensional image of the target.

As we can observe from the 3D reconstructed image in Fig. 22, our system (using the CAS-AVA) can clearly

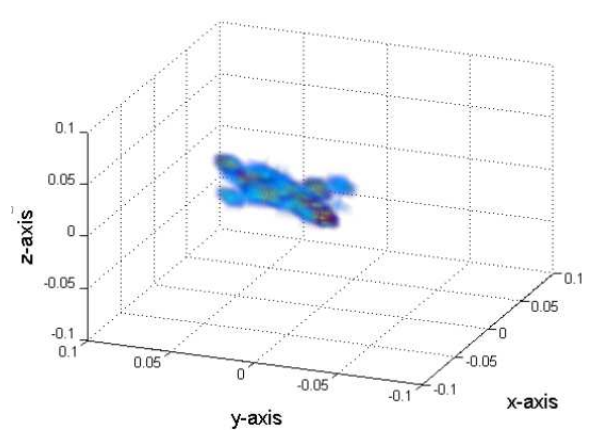

Fig. 22: 3D reconstructed images of the metal target

detect the presence of metallic target with its geometric shape.

\section{Conclusion}

In this paper, a novel compact low profile Antipodal Vivaldi Antenna (AVA) for MIMO imaging system is proposed. A basic AVA has been modified to obtain a larger bandwidth by introducing slots in both sides of the antenna. The simulation results show a good performance in terms of return loss parameter, gain, wide bandwidth and radiation pattern. The proposed antenna is well suited for UWB applications.

We have designed a MIMO array imaging system based on the proposed antenna, in order to confirm its performance. The results show that the proposed antennaCAS-AVA is suitable for our application where it can be easily integrated in the MIMO imaging system enhancing as well the image resolution. As future work, one can target more complex simulation scenarios in terms of topologies for buried object detection applications.

\section{Funding}

This research was supported by the Directorate-General of scientific research and technological development (Project no. 22/Univ.Tlemcen/DGRSDT:

"Development of a buried object detection system using microwave imaging").

Acknowledgements The authors would like to gratefully acknowledge Pr. FEHAM Mohamed, from STIC laboratory of the University of Tlemcen, for his technical support in providing the experimental data. 


\section{Conflict of interest}

On behalf of all authors, the corresponding author states that there is no conflict of interest.

\section{References}

1. S.M. Chouiti, L. Merad, S.M. Meriah, F. Derraz, X. Raimundo, International Journal of Numerical Modelling: Electronic Networks, Devices and Fields 31(5), e2338 (2018). DOI 10.1002/jnm.2338

2. E. Anadol, Ilgin Seker, S. Camlica, Tankut Oguz Topbas, S. Koc, L. Alatan, F. Oktem, O.A. Civi, in Radar Sensor Technology XXII, vol. 10633 (International Society for Optics and Photonics, 2018), vol. 10633, p. 106331D. DOI $10.1117 / 12.2314454$

3. Z. Tahar, X. Derobert, M. Benslama, Progress In Electromagnetics Research C 86, 12 (2018)

4. X. Zhuge, A.G. Yarovoy, IEEE Transactions on Geoscience and Remote Sensing 49(1), 509 (2011). DOI 10.1109/TGRS.2010.2053038

5. G.E. Atteia, A.A. Shaalan, Progress In Electromagnetics Research, PIER 71, 211 (2007)

6. P.J. Gibson, (Brighton, UK, 1979), pp. 101-105

7. E. Gazit, in IEEE Proceeding, Microwave Antennas Propagation, vol. 135 (1988), vol. 135, pp. 89-92

8. E. Hanbay, M.E. Aydemir, in 2019 9th International Conference on Recent Advances in Space Technologies (RAST) (IEEE, Istanbul, Turkey, 2019), pp. 491-495. DOI 10.1109/RAST.2019.8767888

9. A. Gorai, A. Karmakar, M. Pal, R. Ghatak, AEU - International Journal of Electronics and Communications 69(9), 1328 (2015). DOI 10.1016/j.aeue.2015.05.017

10. N.S.B. Hasim, K.A.H. Ping, M.T. Islam, M.Z. Mahmud, S. Sahrani, D.A.A. Mat, D.N.A. Zaidel, Progress In Electromagnetics Research M 80, 35 (2019). DOI 10.2528/PIERM18121201

11. G. Pandey, H. Verma, M. Meshram, Electronics Letters 51(4), 308 (2015). DOI 10.1049/el.2014.3540

12. A.M. De Oliveira, M.B. Perotoni, S.T. Kofuji, J.F. Justo, IEEE Antennas and Wireless Propagation Letters 14, 1334 (2015). DOI 10.1109/LAWP.2015.2404875

13. M. Moosazadeh, S. Kharkovsky, Z. Esmati, B. Samali, in 2016 IEEE-APS Topical Conference on Antennas and Propagation in Wireless Communications (APWC) (2016), pp. 102-105. DOI 10.1109/APWC.2016.7738131

14. A.M.d. Oliveira, J.F. Justo, M.B. Perotoni, S.T. Kofuji, A.G. Neto, R.C. Bueno, H. Baudrand, Microwave and Optical Technology Letters 59(2), 337 (2017). DOI 10.1002/mop.30293

15. M. Moosazadeh, S. Kharkovsky, J.T. Case, B. Samali, Microwave and Optical Technology Letters 59(6), 1259 (2017). DOI 10.1002/mop.30509

16. C.M. Studio, CST Inc. (2014)

17. M. Mighani, M. Akbari, Progress In Electromagnetics Research C 52, 153 (2014). DOI 10.2528/PIERC14053002

18. A. Mehdipour, K. Mohammadpour-Aghdam, R. FarajiDana, Progress In Electromagnetics Research 77, 85 (2007). DOI 10.2528/PIER07072904

19. G. Quintero, J. Zurcher, A.K. Skrivervik, IEEE Transactions on Antennas and Propagation 59(7), 2502 (2011). DOI 10.1109/TAP.2011.2152322
20. Hongsan Sheng, P. Orlik, A. Haimovich, L. Cimini, Jinyun Zhang, in IEEE International Conference on Communications, 2003. ICC '03., vol. 1 (IEEE, Anchorage, AK, USA, 2003), vol. 1, pp. 738-742. DOI 10.1109/ICC.2003.1204271 